\title{
Instability statistics and mixing rates
}

\author{
Roberto Artuso $^{1,2, *}$ and Cesar Manchein ${ }^{1,3, \dagger}$ \\ ${ }^{1}$ Center for Nonlinear and Complex Systems and Dipartimento di Fisica e Matematica, \\ Università degli Studi dell'Insubria, Via Valleggio 11, 22100 Como, Italy \\ ${ }^{2}$ I.N.F.N. Sezione di Milano, Via Celoria 16, 20133 Milano, Italy \\ ${ }^{3}$ Departamento de Física, Universidade Federal do Paraná, 81531-980 Curitiba, Paraná, Brazil \\ (Received 3 June 2009; revised manuscript received 7 August 2009; published 21 September 2009)
}

\begin{abstract}
We claim that looking at probability distributions of finite time largest Lyapunov exponents, and more precisely studying their large deviation properties, yields an extremely powerful technique to get quantitative estimates of polynomial decay rates of time correlations and Poincaré recurrences in the-quite-delicate case of dynamical systems with weak chaotic properties.
\end{abstract}

DOI: $10.1103 /$ PhysRevE.80.036210

PACS number(s): 05.45.Ac

\section{INTRODUCTION}

A general problem of dynamical systems theory concerns both the evaluation of time or space averages, and an understanding of how finite order estimates of such averages converge to their asymptotic limit. The second feature is tightly connected to a quantitative estimation of mixing rates, that is how time correlations asymptotically decay. Though in extremely simplified models (simple Markov chains, lattice models with a finite transition matrix) the connection between convergence of finite order estimates and mixing properties may be established quite easily, the issue in more general contexts is much more complicated, and many facets of the problem still remain as open problems. In particular when weakly chaotic systems are considered it is expected that sticking to regular structures in the phase space severely degrades mixing properties, and critical, polynomial decay of temporal correlations can be observed. We remark that mixed systems are thought to be generic [1] and that slow polynomial decay may influence deeply deterministic transport properties [2], as the Kubo formula suggests. We here propose to look at large deviation properties of finite time estimates as an efficient tool to get quantitative information about mixing properties of the system. We emphasize that the idea of a relationship between the tails of finite time distributions and memory effects is not new: for instance inspection of the tails of finite time Lyapunov exponents was used to get informations about qualitative changes in the dynamics of coupled maps in [3], the detection of small regular islands in $[4,5]$, or motion of a particle in a random time-dependent potential [6] (see also [7] for an example in hyperbolic dynamics). A rigorous analysis, inspiring the present work, was presented in [8,9]: originally [8] a class of one-dimensional maps $f$ was taken into account (the theoretical analysis was extended to higher dimensional systems in [10]: if we denote by $\lambda$ the Lyapunov exponent, and by $P_{n}\left(\lambda_{n}\right)$ the distribution of finite time estimates

\footnotetext{
*roberto.artuso@uninsubria.it

†cmanchein@gmail.com
}

$$
\lambda_{n}\left(x_{0}\right)=\frac{1}{n} \ln \left|\frac{d f^{(n)}(x)}{d x}\right|_{x_{0}} \mid
$$

then, by fixing a threshold $\tilde{\lambda}$ such that $0<\tilde{\lambda}<\lambda$ we may estimate the fraction of initial conditions yielding an estimate below the threshold

$$
\mathcal{M}_{\bar{\lambda}}^{\tilde{n}}(n)=\int_{-\infty}^{\tilde{\lambda}} d \lambda_{n} P_{n}\left(\lambda_{n}\right) .
$$

The quantity $\mathcal{M}_{\hat{\lambda}}(n)$ asymptotically vanishes if the system is ergodic (as we always suppose): for weakly chaotic system it may however decay polynomially,

$$
\mathcal{M}_{\lambda}(n) \sim \frac{1}{n^{\xi}},
$$

in such a case a bound is proven [8] for correlation decay,

$$
\mathcal{C}(n) \leq \frac{1}{n^{\xi-1}},
$$

where, as usual,

$$
\mathcal{C}(n)=\int d \mu(x) \phi(x) \psi\left(f^{(n)}(x)\right)
$$

where $\phi$ and $\psi$ are taken in a suitable class of smooth observables [11] and $\mu$ is the invariant measure of the system. Actually in [8] $\mathcal{M}_{\bar{\lambda}}(n)$ is defined in a slightly different way (a full equivalence is established only via further assumptions): our definition accomplishes a twofold purpose: on the one side it allows a comparison with large deviation estimates in [9], and on the other side it yields an easily computable quantity.

This actually leads to the main point of our paper: we argue that scrutinizing the way in which $\mathcal{M}_{\bar{\lambda}}^{\tilde{\lambda}}(n)$ decays provides an extremely efficient way of studying quantitatively the decay of correlations, a key issue in the analysis of fully and weakly chaotic dynamical systems. As a matter of fact direct quantitative estimates of mixing rates are known to be quite delicate numerically [12], and many efforts have been devoted to devise how to tackle the problem by alternative 
approaches, such as return time statistics [12-15], which can be rigorously shown to yield the correct answer in $1 d$ intermittency [16].

The plan of the paper is as follows: first we suggest, in view of recent results [9], that estimate (4) is not optimal, and the decay properties of $\mathcal{M}_{\hat{\lambda}}^{\tilde{\lambda}}(n)$ and $\mathcal{C}(n)$ are the same; then we check such a conjecture for a class of onedimensional intermittent maps, where polynomial decay rates of correlations are known exactly. We then consider an intermittent area-preserving map, which is a prototype example of intermittency in higher dimensions. We finally reinvestigate, by this technique, the problem of generic correlation decay in maps with a mixed phase space: in all cases the results are coherent with exact results, and corroborate proposed conjectures, in a clean, controlled way.

\section{LARGE DEVIATIONS AND CORRELATION FUNCTION}

The way in which we defined $\mathcal{M}_{\lambda}^{-}(n)$ (2) is in the form of a large deviation result [17]: a remarkable connection between large deviations properties and correlation decay was proven in [9] (see also [18]): if we consider a system for which correlation of smooth functions decay polynomially (the smoothness is essential [11], see also [19]) $\mathcal{C}(n) \sim n^{-\xi}$ then the following results hold, for (Césaro) finite time averages of an observable $\phi$ :

$$
\mu\left(x|| n^{-1} \sum_{k=0}^{n-1} \phi\left(f^{(k)}(x)\right)-\bar{\phi} \mid>\epsilon\right) \leq C_{\phi, \epsilon} \frac{1}{n^{\xi}}
$$

$\mu$ being again the invariant measure, while $\bar{\phi}$ denotes the phase average of the observable. In one dimension, by taking $\phi(x)=\ln \left|f^{\prime}(x)\right|$ this suggests that bound (4) could be sharpened, in such a way that correlation decay and large deviations are characterized by the same power-law exponent: on the other side in dimensions higher than one finite estimates of Lyapunov exponents (computed for instance by selecting the largest eigenvalue of the product of jacobians along a trajectory) lack composition structure of Césaro sums. Our claim is that, despite these provisos, the asymptotic decay of $\mathcal{M}_{\lambda}^{\tilde{\lambda}}(n)$ quantitatively reproduces exactly the decay of correlations, and inspection of such a quantity provides an excellent tool to investigate the weak-chaos regime.

Before applying such a method to a number of dynamical systems, we point out that the way in which we consider the exponent $\xi$ is as the lowest possible value in a suitable class of (zero mean) smooth functions: it is well known that specific choices of observables may yield faster decay (see [20] for a rigorous example in one-dimensional intermittent dynamics).

\section{A. One-dimensional system: the Pikovsky map}

Our first benchmark tool is represented by Pikovsky map [21] $T_{z}$, which is implicitly defined by

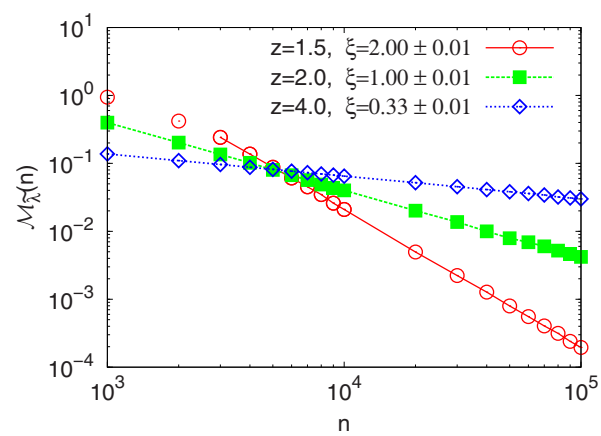

FIG. 1. (Color online) Numerical data (symbols), and regression fits (lines) of power-law decay of $\mathcal{M}_{\lambda}(n)$ for Pikovsky map for three different values of the intermittency exponent. The fit for the case $z=1.5$ starts at $n=3000$. Theoretical values for $\xi$, from Eq. (7), are $\xi=2, \xi=1$, and $\xi=1 / 3$.

$$
x=\left\{\begin{array}{ll}
\frac{1}{2 z}\left[1+T_{z}(x)\right]^{z}, & 0<x<1 /(2 z) \\
T_{z}(x)+\frac{1}{2 z}\left[1-T_{z}(x)\right]^{z}, & 1 /(2 z)<x<1
\end{array},\right.
$$

while for negative values of $x$, the map is defined as $T_{z}(-x)=-T_{z}(x)$ [21]. Such a map has remarkable features, as in $x= \pm 1$ two marginal fixed points of the PomeauManneville type are present (and $z$ is the corresponding intermittency parameter), while for any value of $z$ the invariant measure is Lebesgue [21], as it can be seen by direct inspection of the Perron-Frobenius operator. For this map the correlations decay polynomially, with an exponent that depends upon the intermittency parameter $z$ [22],

$$
\mathcal{C}(n) \sim n^{-1 /(z-1)} .
$$

This decay is very well reproduced by numerical simulations for $\mathcal{M}_{\bar{\lambda}}^{\tilde{n}}(n)$, as we see from Fig. 1. We remark that the numerical simulations for the Pikovsky map were performed by using $10^{6}$ initial conditions uniformly chosen in the phase space $[-1,1]$.

When we want to check how well correlation decay is reproduced by large deviations, expressed by the decay of $\mathcal{M}_{\tilde{\lambda}}^{\tilde{n}}(n)$, we have to be careful to take the large deviation parameter $\tilde{\lambda}$ sufficiently far from the center of the distribution: even if the general expectation is the asymptotic decay law will not depend upon the cutoff, finite time estimates require that $\mathcal{M}$ picks up only contributions in the tail of the distribution. As a matter of fact any choice of the cutoff $\tilde{\lambda}$ will determine a time scale $\tau_{\lambda}^{\sim}$ such that only after such a transient the asymptotic decay is reproduced correctly. This is well illustrated in Fig. 2 where were chosen three values of $\tilde{\lambda}$ and we obtained the asymptotic decay for all cases, but with different time scale $\tau_{\lambda}^{\sim}$.

In Fig. 3 we show the shape (bimodal) of (normalized) finite time distribution $P_{n}\left(\lambda_{n}\right)$ for map (6): as ergodicity predicts, they tend to a Dirac $\delta$ centered on $\lambda$ in the asymptotic limit, but the tails are polynomially "fat" for finite times. In the inset (inside Fig. 3) we can detect an interesting feature: 


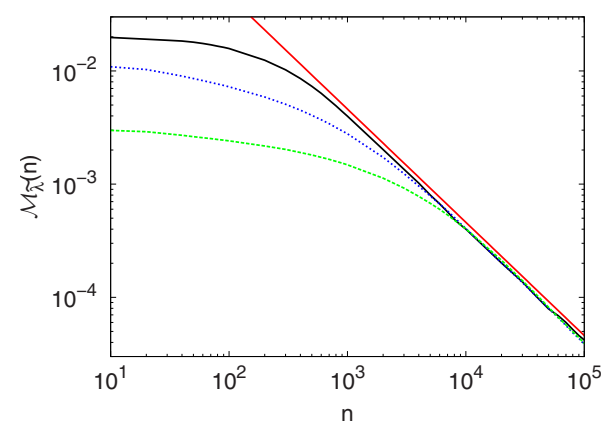

FIG. 2. (Color online) Decay of $\mathcal{M}_{\lambda}^{\sim}(n)$ for system (6) with $z=2.0$, for different cutoffs $\tilde{\lambda}$, where the red (gray) curve is a regression fit of power-law decay with exponent $\xi=1$.

by analyzing the distribution of Lyapunov exponents closer to zero in logarithmic scale it is possible see how the distribution obeys a power-law decay with time.

As a final remark we point out that, though large deviation estimates such as Eq. (5) are symmetric with respect to the asymptotic phase average, from a physical point of view it is quite natural to focus the interest on the small instability branch, as it is precisely anomalous proliferation of almost stable segments of trajectories that induces transition from exponential to power-law decay of temporal correlations.

\section{B. Two-dimensional systems: a family of area-preserving maps}

The second dynamical system we consider is a family of area-preserving maps on the two torus $[-\pi, \pi)^{2}$, depending on two parameters $\varepsilon$ and $\gamma$,

$$
M_{(\varepsilon, \gamma)}:\left\{\begin{array}{ll}
y_{n+1}=y_{n}+f\left(x_{n}\right) & \bmod 2 \pi \\
x_{n+1}=x_{n}+y_{n+1} & \bmod 2 \pi
\end{array},\right.
$$

where $f\left(x_{n}\right)$ is defined by

$$
f\left(x_{n}\right)=\left[x_{n}-(1-\varepsilon) \sin \left(x_{n}\right)\right]^{\gamma} .
$$

A map of this family was introduced in [23], and different features were analyzed in [24-27]: we recall a few of the relevant properties. When $\varepsilon>0$ the map is hyperbolic, while for $\varepsilon=0$ the fixed point at $(0,0)$ becomes parabolic: in such a case dynamics close to the fixed point depends on the value

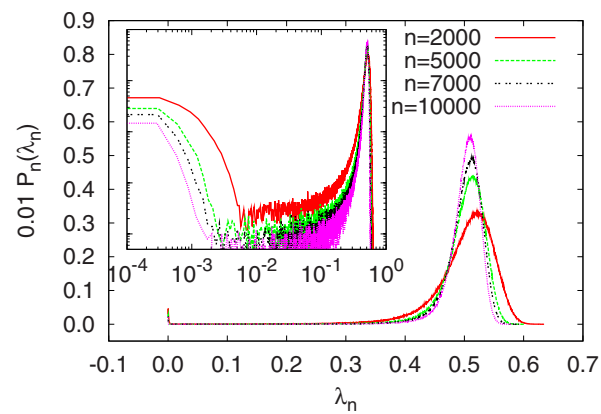

FIG. 3. (Color online) Distribution of Lyapunov exponents $P_{n}\left(\lambda_{n}\right)$ for map (6) with $z=2.0$. Inset: a magnification of $P_{n}\left(\lambda_{n}\right)$ is shown in logarithmic scale. The distribution of Lyapunov exponents closer to zero show a polynomial decay with time.

of $\gamma$, which plays the role of an intermittency parameter: correspondingly the decay of correlations is exponential in the former case, while in the latter a power law is expected, with an exponent depending on $\gamma$. In [27] the following law was proposed:

$$
\mathcal{C}(n) \sim n^{-3(\gamma+1) /(3 \gamma-1)}
$$

in the case $\gamma=1$ a rigorous bound is proven [26] $|\mathcal{C}(n)| \leq n^{-2}$ [while Eq. (10) predicts an exponent -3 ].

Our numerical experiments encompass both regimes: in Fig. 4(a) a hyperbolic parameter choice leads to an exponential decay rate for $\mathcal{M}_{\lambda}^{-}(n)$, while in the intermittent case a power law is observed, with an exponent in agreement with Eq. (10) [see Fig. 4(b)]. In this case the numerical results were obtained by using $10^{6}$ and $10^{8}$ initial conditions uniformly distributed in the phase space, respectively.

The case reported in Fig. 4(b) allows to scrutinize the numerical virtues of our approach with alternative methods (that were employed in [27]): it provides estimates as sharp as the analysis of return time statistics (by employing however much less initial conditions), while it outperforms direct computation of correlations.

\section{Ensemble of modified standard maps}

The last case we present concerns a general issue, namely whether universality properties are exhibited for Hamiltonian systems with a hierarchical (mixed) phase space. This is a
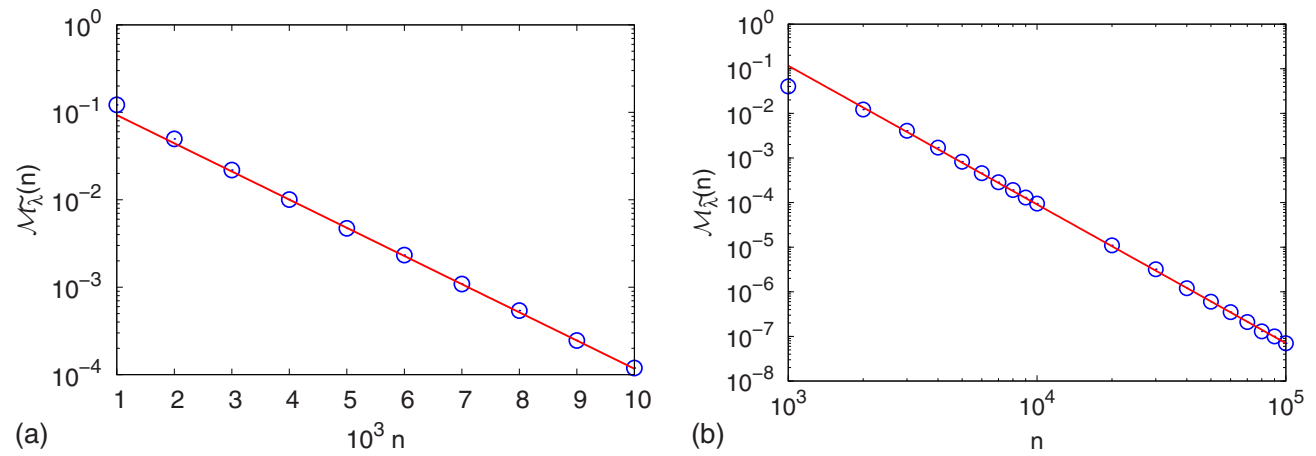

FIG. 4. (Color online) Decay $\mathcal{M}_{\lambda}^{\sim}(n)$ (symbols) together with a regression fit (full lines) for map (8) with (a) $\varepsilon=0.5$ and $\gamma=1$ (exponential rate decay $0.87 \pm 0.01$ ) and (b) $\varepsilon=0$ and $\gamma=1$ (polynomial rate decay $3.05 \pm 0.05$ ). Both fits were done by starting at $n=3000$. 
much debated issue (we refer to [28,29] for relevant references over the last twenty years): in particular [28] suggests, on the basis of a Markov tree model [30] with random scaling factors for transition probabilities, the existence of an asymptotic algebraic decay for the Poincaré recurrences. The authors also discuss in detail the numerical difficulties in probing such a claim: in particular, following [31,32] they advocate that an average over different Hamiltonian systems significantly reduces the extremely long times that are required for a single trajectory to sample fine details of the phase-space structure: this procedure allows them to check for an ensemble of area-preserving maps their proposal, and the polynomial decay exponent of Poincaré recurrences is estimated as $\chi \simeq 1.57 \pm 0.03$, which, according to [12-15], suggests for correlations the decay law

$$
\mathcal{C}(n) \sim \frac{1}{n^{\chi^{-1}}}
$$

The exponent of algebraic decay of Poincaré recurrences was also numerically investigated in [29] (with an estimate $\chi \simeq 1.60 \pm 0.05$ ), for a different ensemble of area-preserving maps, which we also utilize in the present framework.

The ensemble of modified standard maps on $[-\pi, \pi)^{2}$ considered here are given by

$$
P_{K, K^{\dagger}}:\left\{\begin{array}{l}
p_{n+1}=p_{n}+K \sin \left(x_{n}\right)+K^{\dagger} \\
x_{n+1}=x_{n}+p_{n+1}
\end{array},\right.
$$

where $K$ and $K^{\dagger}$ are the nonlinear parameter and magnetic field, respectively. They are uniformly chosen in the intervals $K \in[\pi, 1.2 \cdot \pi]$ and $K^{\dagger} \in[0.0,0.4 \cdot \pi]$. We have used $10^{6}$ initial conditions taken uniformly in the square $x_{0} \in[-0.1,0.1], p_{0} \in[-0.1,0.1]$, well inside the chaotic region. The behavior we show in Fig. 5 apparently arises after a few $(\sim 10)$ random realizations of the maps: the overall decay rate with an exponent $0.57 \pm 0.05$ is stable, as well as the oscillations that may be noticed in Fig. 5. Wether such oscillations are due to finite-size effects, corrections to scaling, or multifractality [33], is a feature that in our opinion deserves further investigations. Again we remark that the present method has an important advantage that it needs a "small" number of initial conditions and realizations of the map to show the asymptotic decay of $\mathcal{M}_{\bar{\lambda}}^{\tilde{\lambda}}(n)$ as compared with traditional procedures using direct computation of correlation functions or Poincaré recurrences.

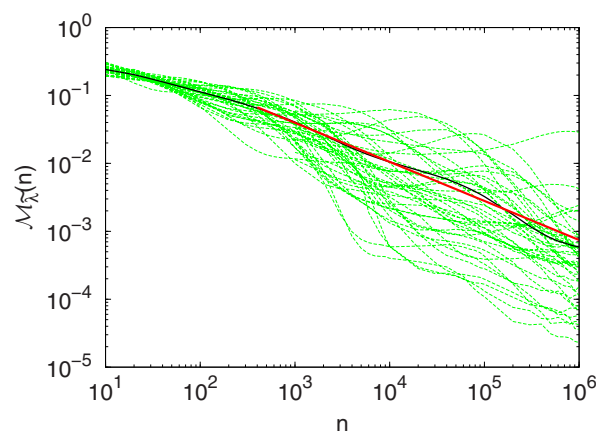

FIG. 5. (Color online) Distribution of largest Lyapunov exponents for 40 realizations of map (12). The black curve is the average over all curves and the red (gray) line corresponds to the fit of average. The fit decay with an exponent $0.57 \pm 0.05$. The fit was done by starting at $n=500$.

\section{CONCLUSIONS}

We have shown how the analysis of large deviations for Lyapunov exponents provides a very efficient way to investigate correlation and Poincaré recurrences decay for dynamical systems: in particular when dealing with weakly chaotic systems, with associated polynomial correlation decay, the power-law exponent has been computed from the distribution function of finite time Lyapunov exponents with remarkable accuracy.

We also remark that in all our tests this procedure performs quite well numerically: for instance in our last example the estimate has been obtained with a computational effort significantly reduced with respect to former simulations $[28,29]$. Another interesting point we plan to investigate in the future is to study the decay law of correlation function in higher dimensional systems where the nonlinearity is not uniformly distributed along different unstable directions [34].

\section{ACKNOWLEDGMENTS}

One of the authors (C.M.) gratefully acknowledges CNPq Brazil for support and the Center for Nonlinear and Complex Systems in Como for hospitality. We thank Marcus W. Beims, Giulio Casati, Giampaolo Cristadoro, Stefano Luzzatto, Matteo Sala, and Sandro Vaienti for very useful correspondence and discussions.
[1] L. Markus and K. R. Meyer, Mem. Am. Math. Soc. 144, 1 (1974).

[2] R. Artuso and G. Cristadoro, in Anomalous Transport: Foundations and Applications, edited by R. Klages, G. Radons, and I. M. Sokolov (Wiley-VCH, New York, 2008).

[3] M. Falcioni, U. Marini Bettolo Marconi, and A. Vulpiani, Phys. Rev. A 44, 2263 (1991).

[4] S. Tomsovic and A. Lakshminarayan, Phys. Rev. E 76, 036207 (2007).
[5] M. W. Beims, C. Manchein, and J. M. Rost, Phys. Rev. E 76, 056203 (2007).

[6] H. Schomerus and M. Titov, Phys. Rev. E 66, 066207 (2002).

[7] C. Anteneodo, Phys. Rev. E 69, 016207 (2004).

[8] J. F. Alves, S. Luzzatto, and V. Pinheiro, Ergod. Theory Dyn. Syst. 24, 637 (2004).

[9] I. Melbourne, Proc. Am. Math. Soc. 137, 1735 (2009).

[10] J. F. Alves, S. Luzzatto, and V. Pinheiro, Ann. Inst. Henri Poincare, Sect. A 22, 817 (2005). 
[11] J. D. Crawford and J. R. Cary, Physica D 6, 223 (1983).

[12] R. Artuso, Physica D 131, 68 (1999).

[13] S. R. Channon and J. L. Lebowitz, Ann. N.Y. Acad. Sci. 357, 108 (1980).

[14] B. V. Chirikov and D. L. Shepelyansky, Physica D 13, 395 (1984).

[15] C. F. F. Karney, Physica D 8, 360 (1983).

[16] L.-S. Young, Isr. J. Math. 110, 153 (1999).

[17] F. den Hollander, Large Deviations (AMS, Brooklyn, NY, 2000).

[18] M. Pollicott and R. Sharp, Nonlinearity 22, 2079 (2009).

[19] P. Collet and S. Isola, Commun. Math. Phys. 139, 551 (1991).

[20] S. Gouëzel, Isr. J. Math. 139, 29 (2004).

[21] A. S. Pikovsky, Phys. Rev. A 43, 3146 (1991).

[22] G. Cristadoro, N. Haydn, P. Marie, and S. Vaienti, e-print arXiv:0812.0555.

[23] J. Lewowicz, J. Differ. Equations 38, 192 (1980).

[24] R. Artuso and A. Prampolini, Phys. Lett. A 246, 407 (1998).

[25] C. Liverani, Math. Phys. Electron. J. 10, 1 (2004).
[26] C. Liverani and M. Martens, Commun. Math. Phys. 260, 527 (2005).

[27] R. Artuso, L. Cavallasca, and G. Cristadoro, Phys. Rev. E 77, 046206 (2008).

[28] G. Cristadoro and R. Ketzmerick, Phys. Rev. Lett. 100, 184101 (2008).

[29] E. G. Altmann, Ph.D. thesis, Max Planck Institut für Physik Komplexer Systeme, 2007 (unpublished).

[30] J. D. Meiss and E. Ott, Phys. Rev. Lett. 55, 2741 (1985).

[31] B. V. Chirikov and D. L. Shepelyansky, in Proceedings of the IXth International Conference on Nonlinear Oscillations (Naukova Dumka, Kiev, UIkraine, 1981), Vol. 2, p. 420; [Princeton University Report No. PPPL-TRANS-133, 1983 (unpublished)].

[32] F. Christiansen and P. Grassberger, Phys. Lett. A 181, 47 (1993).

[33] V. Afraimovich and G. M. Zaslavsky, Phys. Rev. E 55, 5418 (1997).

[34] C. Manchein, M. Beims, and J. Rost, e-print arXiv:0907.4181. 